\title{
Intrathyroidal Epithelial Thymoma: Carcinoma Showing Thymus-like Differentiation Mimicking Squamous Cell Carcinoma of the Thyroid
}

\author{
Aya Ebina ${ }^{1}$, Iwao Sugitani ${ }^{2}$ and Noriko Motoi ${ }^{3,4}$ \\ ${ }^{1}$ Division of Head and Neck, Cancer Institute Hospital, Japanese Foundation for Cancer Research \\ ${ }^{2}$ Department of Endocrine Surgery, Nippon Medical School \\ ${ }^{3}$ Department of Pathology, Cancer Institute Hospital, Japanese Foundation for Cancer Research \\ ${ }^{4}$ Division of Pathology, the Cancer Institute, Japanese Foundation for Cancer Research
}

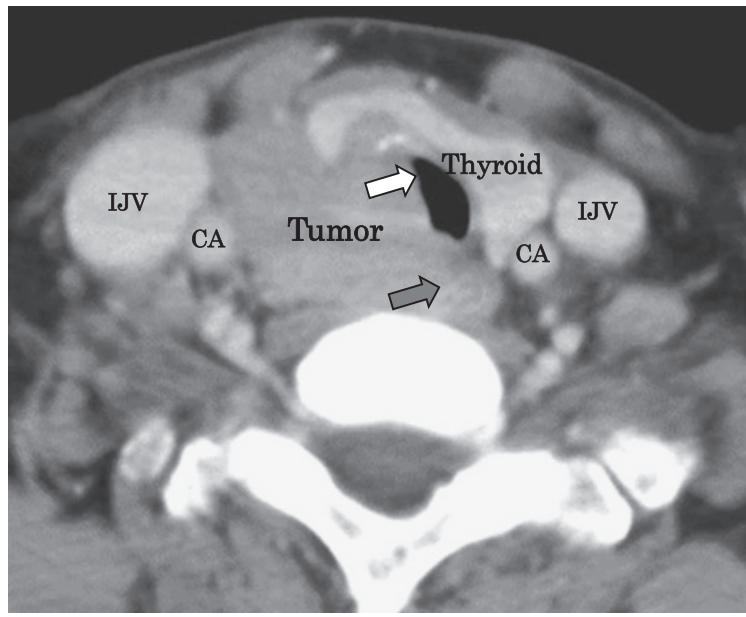

Fig. 1

Carcinoma of possible thymic epithelial origin may occur within the thyroid gland and was first reported by Miyauchi et al. ${ }^{1}$ as intrathyroidal epithelial thymoma (ITET) in 1981. Ten years later, Chan and Rosai called this neoplasm carcinoma showing thymus-like differentiation (CASTLE) and described its clinical and pathological features ${ }^{2}$. ITET/CASTLE is a rare malignant tumor having histopathological features similar to those of squamous cell carcinoma (SCC) of the thyroid. Primary SCC of the thyroid usually has a dismal prognosis comparable to that of anaplastic thyroid carcinoma (ATC); however, the prognosis of ITET/CASTLE is more favorable. Curative resection followed by radiation therapy may effectively prevent locoregional recurrence. ${ }^{3}$. Immunohistochemical staining with CD5, a marker of carcinoma of thymic origin, is helpful for diagnosing ITET/ CASTLE ${ }^{4}$. We report a case of ITET/CASTLE mimicking ATC with a SCC component.

A 63-year-old woman with a 6-month history of dyspnea was found to have a mass of the right side of the neck. Cross-sectional imaging showed a 5-cm right thyroid tumor that had invaded the trachea and esophagus (Fig. 1). Metastasis to the lymph nodes or lungs was not clinically evident. The findings of fine-needle aspiration biopsy were suggestive of ATC. Subsequently, the patient underwent total pharyngolaryngectomy, near-total thyroidectomy with bilateral neck dissection, and reconstruction of the cervical esophagus with a free jejunum flap. After surgery, she was treated with chemoradiotherapy (40 Gy external radiation combined with doxorubicin, cisplatin, and fluorouracil). The postoperative histological diagnosis was ATC with a squamous-cell component; however, she survived 10 years after treatment without recurrence. Re-evaluation of the histologic diagnosis with immunostaining for CD5 revealed the actual diagnosis to be ITET/CASTLE (Fig. 2).

Conflict of Interest: The authors declare no conflict of interest.

Correspondence to Iwao Sugitani, MD, PhD, Department of Endocrine Surgery, Nippon Medical School, 1-1-5 Sendagi, Bunkyo-ku, Tokyo 113-8603, Japan 

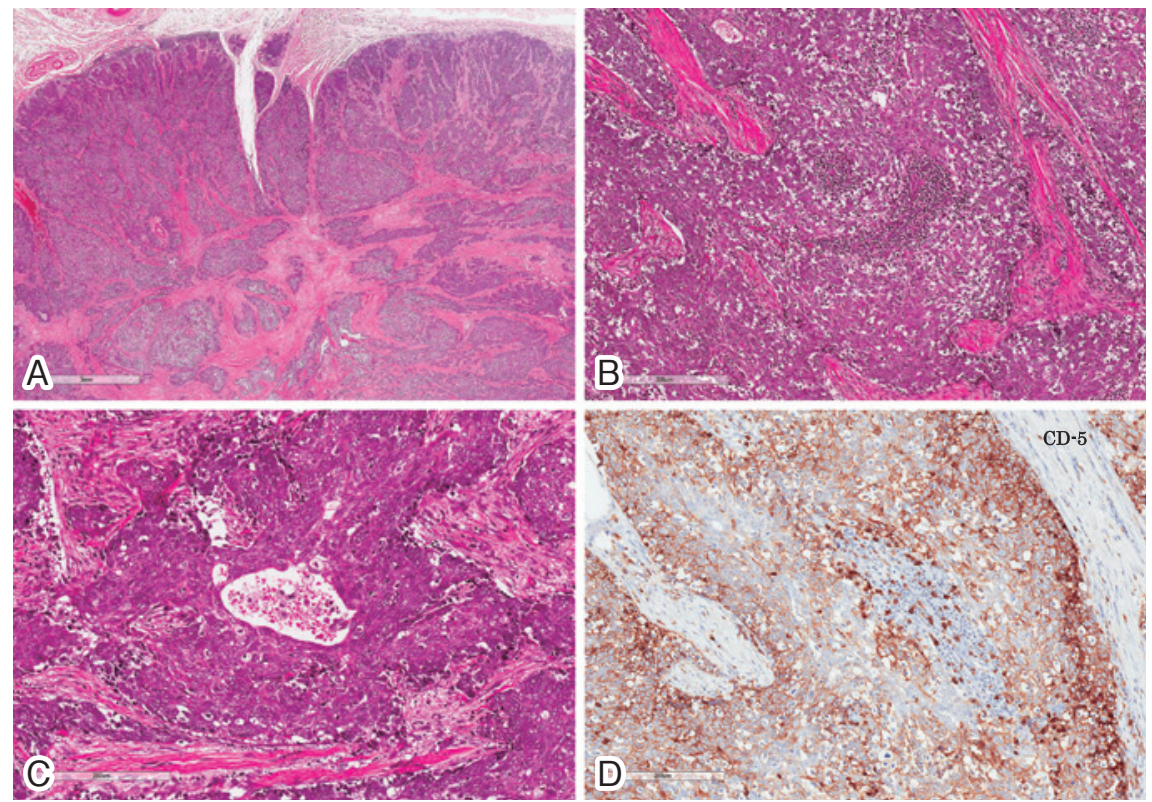

Fig. 2

Fig. 1 Enhanced computed tomography scan of the neck showed a right-sided thyroid mass (tumor) that had invaded the trachea (white arrow) and the esophagus (gray arrow).

Tumor: thyroid tumor invading of the trachea and esophagus. CA: carotid artery. IJV: internal jugular vein.

Fig. 2 Histopathological findings of CASTLE

(A) Tumor showed solid, lobulated, and expanding growth with fibrous stroma and lymphoplasmacytic infiltration. (Bar $=3 \mathrm{~mm}$, original magnification $\times 1.25$ )

(B) Tumor cells were round to short and spindle-shaped with basophilic cytoplasm. The nuclei were round to oval and hyperchromatic with inconspicuous nucleoli. Scattered pyknotic change was also noted. (Bar=0.2 $\mathrm{mm}$, original magnification $\times 20$ )

(C) The presence of central degeneration within the tumor is reminiscent of squamous cell carcinoma. (Bar=0.2 mm, original magnification $\times 20)$

(D) On immunohistochemical examination, tumor cells were positive for cytokeratin AE1/3 and CD5 but negative for thyroglobulin and TTF1. Immunoreactivity for CD5 was also detected in infiltrating $\mathrm{T}$ lymphocytes. (Bar=0.2 $\mathrm{mm}$, original magnification $\times 20$ )

\section{References}

1. Miyauchi A, Kuma K, Matsuzuka F, et al.: Intrathyroidal epithelial thymoma: an entity distinct from squamous cell carcinoma of the thyroid. World J Surg 1985; 9: 128-135.

2. Chan JK, Rosai J, et al.: Tumor of the neck showing thymic or related branchial pouch differentiation: a unify concept. Hum Pathol 1991; 22: 349-367.

3. Ito Y, Miyauchi A, Nakamura Y, et al.: Clinicopathologic significance of intrathyroidal epithelial thymoma/carcinoma showing thymus-like differentiation. A collaborative study with member institutes of the Japanese Society of Thyroid Surgery. Am J Clin Pathol 2007; 127: 230-236.

4. Kakudo K, Bai Y, Ozaki T, et al.: Intrathyroidal epithelial thymoma (ITET) and carcinoma showing thymus-like differentiation (CASTLE). CD5-positive neoplasms mimicking squamous cell carcinoma of the thyroid. Histol Histopathol 2013; 28: 543-556.

E-mail: isugitani@nms.ac.jp Journal Website (http://www.nms.ac.jp/jnms/) 